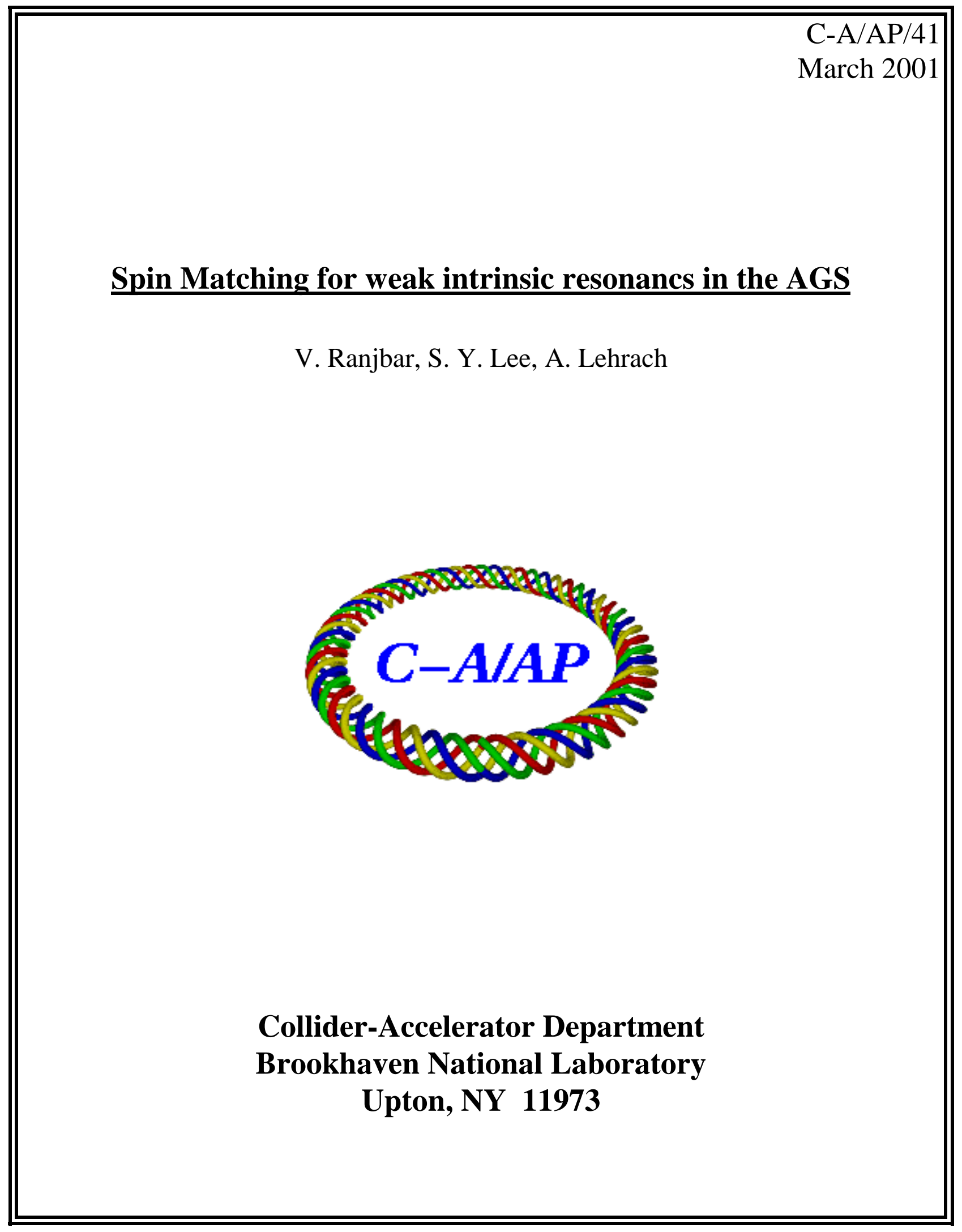




\title{
Spin Matching for weak intrinsic resonances in the AGS
}

\author{
V. Ranjbar ${ }^{1}$, S.Y.Lee ${ }^{2}$, A.Lehrach ${ }^{1}$ \\ ${ }^{1}$ Brookhaven National Laboratory, Upton NY \\ ${ }^{2}$ Indiana University, Bloomington IN
}

March 2, 2001

\begin{abstract}
We examine in detail the use of a family of quadrupoles distributed over each lattice superperiod to effect a reduction of intrinsic resonance strength and further developed the theoretical basis to understand this process. We reconfirm previous results which showed that in order to achieve a complete suppression of these resonances in the AGS can be accomplished with the addition of a single family of quadrupoles[1].We then went further to examine the use of multiple families of quadrupoles. In particular we showed that using tuning quadrupoles already in place in the AGS that a reduction in resonance strength is possible. Finally we proved that a simple linear fit can be used to predict the functional dependence of individual resonances on the integrated field strength of the quadrupoles and developed a full field fit over several possible quadrupole insertions .
\end{abstract}




\section{Depolarization in the AGS}

In the AGS imperfection resonances have been cured using a partial solenoid snake[2] [5], and the strong intrinsic resonances using an rf dipole[3]. However the solenoid snake has introduced a strong linear coupling resonances which still remains to be dealt with as well the weak intrinsic resonances still remain. There are two current methods proposed to deal with the coupling resonances. The simplest but most expensive way is to replace the solenoid snake with a helical dipole snake thus eliminating the coupling solenoid field[4]. The second and more cost effective way proposed is to use a horizontal RF dipole in the fully coupled mode to flip both the intrinsic and coupled resonances simultaneously[6]. This method was investigated in the August 2000 polarized proton run. Results have not proved conclusive, but more work is currently being conducted.

As for the weak intrinsic resonances since it constitutes only $10 \%$ polarization loss not much attention has been paid to this subject. It has been proposed that a modification in the optics of the AGS can theoretically suppress these weak resonances [1] [7]. Dedicated beam time at low energy an thus higher analyzing power will allow for the actual testing of this method. The purpose of this paper to develop the theoretical background necessary to run such an experiment.

\section{Intrinsic Spin Matching.}

Since the strength and location of resonances is tied to the symmetry of each superperiod and the periodic structure of the lattice one strategy to suppress or enhance a resonance is by the careful placement and field strength setting of quadrupole elements. It is well known that in certain instances using carefully placed families of quadrupoles one can break the symmetry of lattice and thus effectively raise the superperiodicity reducing the number of intrinsic resonance 
crossings. Even if a change in the symmetry of the lattice doesn't effect the underlining superperiodicity it is possible to use the quadrupoles to suppress individual resonances by the introduction of a counterveilding perturbation to the resonance strength given by [8]:

$\Delta \varepsilon_{k}=\left(\frac{1+G \gamma}{4 \pi}\right) \sqrt{\frac{\epsilon_{N}}{\pi \gamma}} \int \Delta g(s) \sqrt{\beta_{z}}\left(e^{i\left(\nu_{z} \varphi-k \theta\right)}+e^{-i\left(v_{z} \varphi-k \theta\right)}\right) d s$

We use $\Delta g(s)=\frac{\frac{\partial B_{z}}{\partial x}}{B \rho}$ as the focusing strength, $G$ the anomalous magnetic moment coefficient, $\epsilon_{N}$ the normalized vertical emittance, $\beta_{z}$ the vertical betatron function, $v_{z}$ the vertical tune, $\theta$ the bending angle around the ring and $\varphi$ the betaron phase and $k$ is the resonance tune. This equation will give a good approximation providing that both $\Delta g(s)$ and $\Delta \beta_{z}(s)$ are small. It should be noted that when the spin tune, $G \gamma=k=n P \pm v_{z}$ then an intrinsic resonance is achieved where $\mathrm{P}$ is the superperiodicity of the lattice and $\mathrm{n}$ is any integer. It seems that if we use just the right field strengths and locations we can use this perturbation in the resonance to cancel our existing resonance. This is know as spin matching.

\section{Intrinsic resonance structure in the AGS}

The AGS lattice consits of twelve superperiods each containing twenty combined function magnets of long and short lengths. In Figure 1 you can see graphical representation of the lattice. The resulting structure proves fairly complex. But some general observations are possible. The lattice can be broken down into two sections which are antisymmetric. Further these section can be broken down into a total of four sections of two antisymmetric pairs. There are also two mirror symmetric pairs. While clearly the overriding periodicity of 12 places all our intrinsic resonances at $G \gamma=12 n \pm v_{z}$, the antisymmetric structure can explain the odd and even substructure of the resonances. This explanation 
follows in a manner similar to the evaluation of a typical FODO lattice structure.

Here the focusing and defocusing elements (which are antisymmetric pairs) can contribute additively to the spin kick when the resonance is odd. Applying this logic to the AGS lattice we can see that the middle two antisymmetric pairs can viewed as having an overall sign difference when viewed as a whole. The same is true for the outer two antisymmetric pairs. So depending on how we count there will be an overall phase difference of $\left(k \pm v_{z}\right) \frac{n \pi}{12}$ between at least one antisymmetric pair. Like the evaluation of a typical FODO lattice structure the antisymmetric elements contribute additively to the spin kick when $\mathrm{n}$ is odd and subtract when $\mathrm{n}$ is even . Excluding the $G \gamma=0+v_{z}$ resonance, this explains why we see in Table 1 that the odd resonances are larger than the even ones.

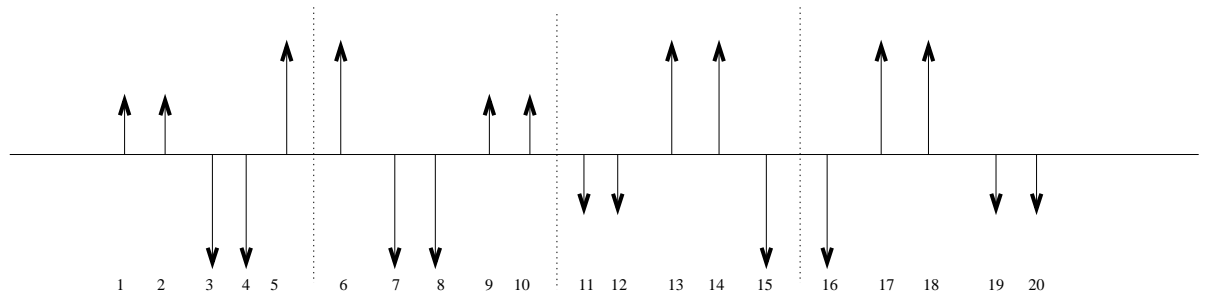

Figure 1:AGS superperiod. Here the up and down vectors show the direction and magnitude of the focusing gradient field. 


\begin{tabular}{|c|c|c|c|c|}
\hline$\gamma$ & $\mathrm{G} \gamma$ & $\operatorname{Re}\left(\varepsilon_{k}\right) 10^{-3}$ & $\operatorname{Im}\left(\varepsilon_{k}\right) 10^{-3}$ & $\left|\varepsilon_{k}\right| 10^{-3}$ \\
\hline \hline 4.871 & $0+v$ & 6.128 & -0.271 & 6.134 \\
\hline 8.515 & $24-v$ & 0.240 & 0.011 & 0.240 \\
\hline 11.565 & $12+v$ & 0.094 & 2.118 & 2.120 \\
\hline 15.208 & $36-v$ & -0.248 & 5.589 & 5.595 \\
\hline 18.258 & $24+v$ & 0.507 & -0.022 & 0.508 \\
\hline 21.902 & $48-v$ & 0.652 & 0.029 & 0.652 \\
\hline 24.951 & $36+v$ & 0.479 & 10.820 & 10.831 \\
\hline
\end{tabular}

Table 1: Resonance strengths $\varepsilon_{k}$ as calculated with DEPOL [9] for the bare AGS machine using a $10 \pi \mathrm{mm}$-mrad normalized emittance. We assume $95 \%$ of the particles are within a gaussian distribution.

The complexity of the lattice seems to prohibit a real increase in the superperiodicty of the lattice with out significant and costly modifications. While there exists many points of overall mirror symmetry, it is clear that either a single or several quadrupoles cannot effect the overall periodicity. If we approach the problem from a purely spin matching aspect then it seems that individual resonances can be suppressed with a well placed family of quadrupoles. Since our resonance structures shows that the $G \gamma=12 n \pm v_{z} \mathrm{n}=$ even resonances are primarily real and the $n=$ odd are primarily imaginary a quadrupole of the right strength and phase location could suppress individual resonances.

\section{Preliminary Calculations for One Family of Quadrupoles}

It might prove insightful if we try to develop an analytical approximation to equation 3.1. If we first consider the effect of only one additional quadrupoles per superperiod, equation 3.1 can be integrated as sum over the number of superperiods in the lattice. This series can be summed using the properties of 
a geometric series.

$$
\begin{gathered}
\Delta \varepsilon_{k}=\left(\frac{1+G \gamma}{4 \pi}\right) \sqrt{\frac{\epsilon_{N}}{\pi \gamma}}\left\{e^{i\left(v_{z}+k\right)\left(\frac{P-1}{P}\right) \pi} \zeta_{P}\left(\frac{k+\nu}{P}\right)\left[g_{1} \sqrt{\beta_{1 z}} e^{i\left(\nu_{z} \varphi_{1}+k \theta_{1}\right)}\right]+\right. \\
\left.e^{i\left(k-v_{z}\right)\left(\frac{P-1}{P}\right) \pi} \zeta_{P}\left(\frac{k-\nu}{P}\right)\left[g_{1} \sqrt{\beta_{1 z}} e_{2}^{i\left(k \theta_{1}-\nu_{z} \varphi_{1}\right)}\right]\right\}
\end{gathered}
$$

Here $\theta_{1}$ and $\varphi_{1}$ are the angle and phase at the quadrupole's position. $\zeta_{P}(x)$ is the enhancement function which for $\mathrm{x}=\mathrm{N}(N=0, \pm 1, \pm 2, \ldots)$ goes to $(-1)^{N} P$ for even $P$ and $P$ for odd $P$. We also assume that we are inserting one quadrupole per superperiod $(\mathrm{P})$. If we pick the location of the quadrupole correctly then we can control either the imaginary or real part of the resonance. So for example in the AGS where $\theta=\varphi$ if we choose the 15 th position which corresponds to $22.5^{\circ}$ then we can control the real part for all $G \gamma=12 n \pm v_{z}$ $n=$ even and the imaginary part for $n=o d d$. In principle provided that there are no other limitations we can suppress these components of the resonance strength to an arbitrary degree. This is exactly what A.Lehrach showed in his paper[1] on suppressing intrinsic spin harmonics. We re-confirmed his results in Figure 2 where the $G \gamma=24 \pm v_{z}$ and $48-v_{z}$ have been successfully suppressed. As well we plotted equation 3.2 on top of the DEPOL generated data. With the inclusion of a $180^{\circ}$ (DEPOL does this by changing the sign on the focusing field strength) initial phase advance our perturbation approximation generates the appropriate structure, though for the $G \gamma=24-v_{z}$ the slope of our line appears significantly off. A detailed comparison between our calculations and DEPOL's algorithm[9] reveal that the discrepancy is due primary to the impact of betatron perturbation on the original lattice structure. The calculated perturbation at each quadrupole proved fairly accurate using equation 3.2. However the remaining elements experience a significant deviation from the bare machine 
values due to perturbations in the betatron function. This question will handled in more detail in the final subsection. Suffice it to say that this model provides an adequate description of the method by which a particular resonance can be suppressed.

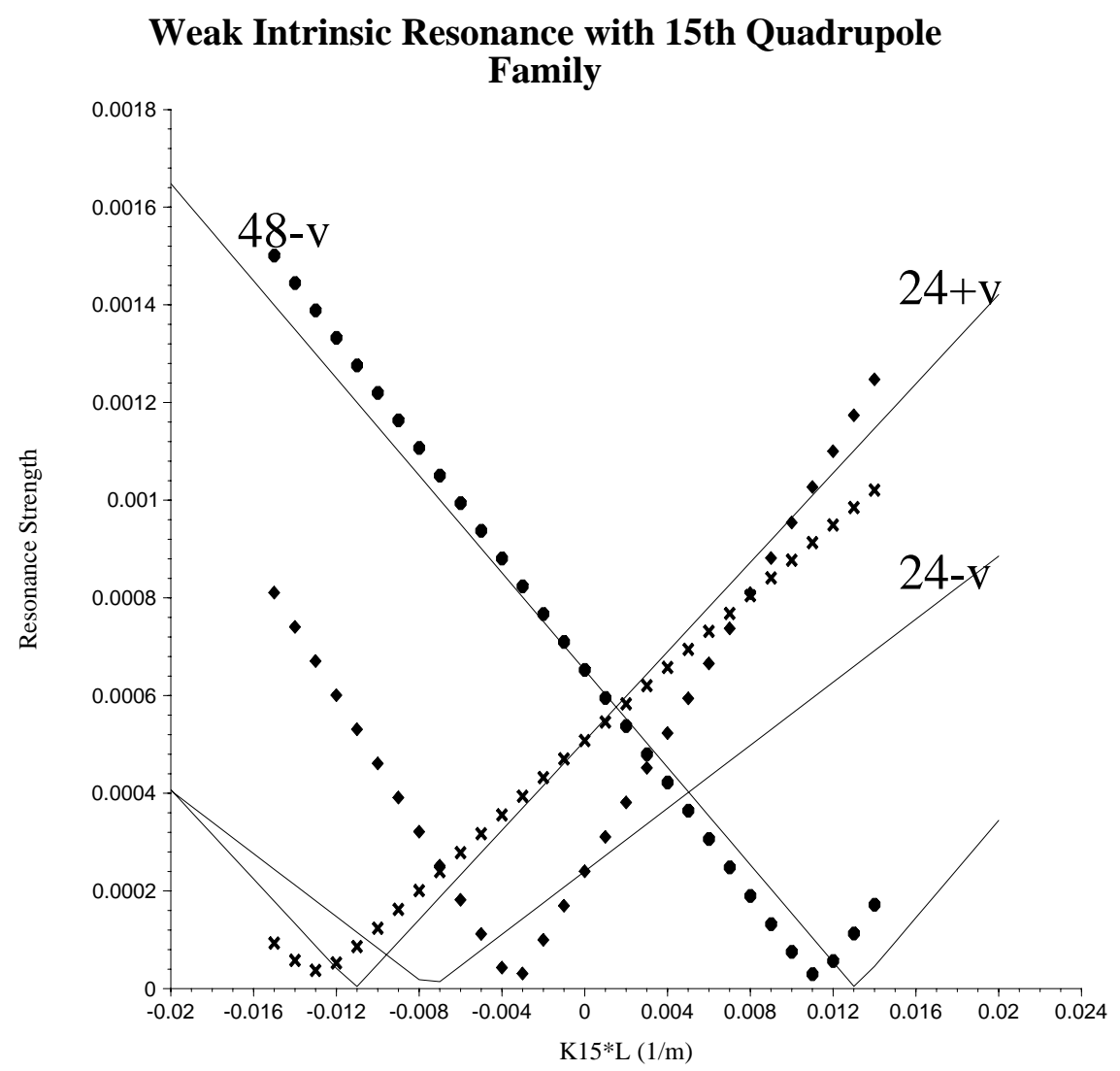


Figure 2: Resonance strength with quadrupole operating in the 15th position. Lines indicate analytical approximation and markers DEPOL generated data.

Attempts to use only quadrupoles located in the 3 rd position failed to completely suppress the same resonances. This is because at $3 \mathrm{rd}$ position $\varphi=4.5^{\circ}$ thus 12 , 24 and 48 yield both imaginary and real resonance contributions. However, if we combine two quadrupoles, the $3 \mathrm{rd}$ and 17 th locations together then we can construct a situation where either the imaginary or real part of our perturbation will cancel exactly leaving only a real or imaginary part.

\section{Preliminary Calculations for Two Families of Quadrupoles}

Developing equation 3.2 further to handle two families of Quadrupoles and writing in terms of $\varphi$ our equation for the shift in resonance strengths becomes (for AGS only).

$$
\begin{aligned}
\Delta \varepsilon_{k}= & \left(\frac{1+G \gamma}{4 \pi}\right) \sqrt{\frac{\epsilon_{N}}{\pi \gamma}}\left\{e^{i\left(v_{z}+k\right)\left(\frac{P-1}{P}\right) \pi} \zeta_{P}\left(\frac{k+\nu_{z}}{P}\right)\left[g_{1} \sqrt{\beta_{1 z}} e^{i\left(\nu_{z}+k\right) \varphi_{1}}+g_{2} \sqrt{\beta_{2}} e^{i\left(\nu_{z}+k\right) \varphi_{2}}\right]+\right. \\
& \left.e^{i\left(k-v_{z}\right)\left(\frac{P-1}{P}\right) \pi} \zeta_{P}\left(\frac{k-\nu_{z}}{P}\right)\left[g_{1} \sqrt{\beta_{1 z}} e^{i\left(k-v_{z}\right) \varphi_{1}}+g_{2} \sqrt{\beta_{2 z}} e^{i\left(k-\nu_{z}\right) \varphi_{2}}\right]\right\}
\end{aligned}
$$

This equation can broken into its real and imaginary parts and simplified by considering only the $K=N P \pm \nu$ where $\mathrm{N}$ is a positive integer.

$$
\operatorname{Re}(\Delta \varepsilon)=\left(\frac{1+G \gamma}{4 \pi}\right) \sqrt{\frac{\epsilon_{N}}{\pi \gamma}} P(-1)^{N P}\left(g_{1} \sqrt{\beta_{1 z}} \cos \left(N P \varphi_{1}\right)+g_{2} \sqrt{\beta_{2 z}} \cos \left(N P \varphi_{2}\right)\right)
$$


$\operatorname{Im}(\Delta \varepsilon)=\left(\frac{1+G \gamma}{4 \pi}\right) \sqrt{\frac{\epsilon_{N}}{\pi \gamma}} P(-1)^{N P}\left(g_{1} \sqrt{\beta_{1 z}} \sin \left(N P \varphi_{1}\right)+g_{2} \sqrt{\beta_{2 z}} \sin \left(N P \varphi_{2}\right)\right)$

If we assume that equation 3.3 constitutes a linear perturbation to the original resonance strength then an approximate solution for the zero resonance case can be easily developed. For example if we are interested in only the even resonances which are predominately real then we can solve for $g_{1}$ and $g_{2}$.

$$
\begin{gathered}
g_{1}=-g_{2} \sqrt{\frac{\beta_{2 z}}{\beta_{1 z}}} \frac{\sin \left(N P \varphi_{2}\right)}{\sin \left(N P \varphi_{1}\right)} \\
g_{2}=\frac{-R_{0} 4 \pi}{(1+G \gamma) \sqrt{\frac{\epsilon_{N}}{\pi \gamma}} P \sqrt{\beta_{2 z}}\left[\cos \left(N P \varphi_{2}\right)-\sin \left(N P \varphi_{2}\right) \cot \left(N P \varphi_{1}\right)\right]}
\end{gathered}
$$

In Figure 3-4 we can see the $G \gamma=48-v_{z}$ resonance calculated via DEPOL and using equations 3.3-3.6 . Again using an $180^{\circ}$ phase advance our approximation while agreeing that our resonance can be completely suppressed, fails to locate its position in $g_{1}$ and $g_{2}$ space. Never-the-less it is clear that using the 3rd and 17th quadrupoles simultaneously a complete suppression is possible in theory.

Unfortunately in reality we are limited in the field strength values available to us by the following factors:

1.The integer and half integer tune stop-bands.

2.The speed and strength which the quadrupoles can ramp the field.

In fact to completely suppress the $G \gamma=48-v_{z}$ resonance forces us to tunes well below the half integer. Given these limitations we limit ourselves to the problem of weak intrinsic resonance suppression, because the strong resonance require both a field strength beyond the capacity of our current quadrupoles 
and more problematic would require crossing the integer and half integer tunes. In light of these factors it would be instructive to consider in detail the relationship between the quadrupole field strength and tune as well as expand our understanding of the impact the quadrupoles have on the betatron function.

\section{8-v resoance approximation}

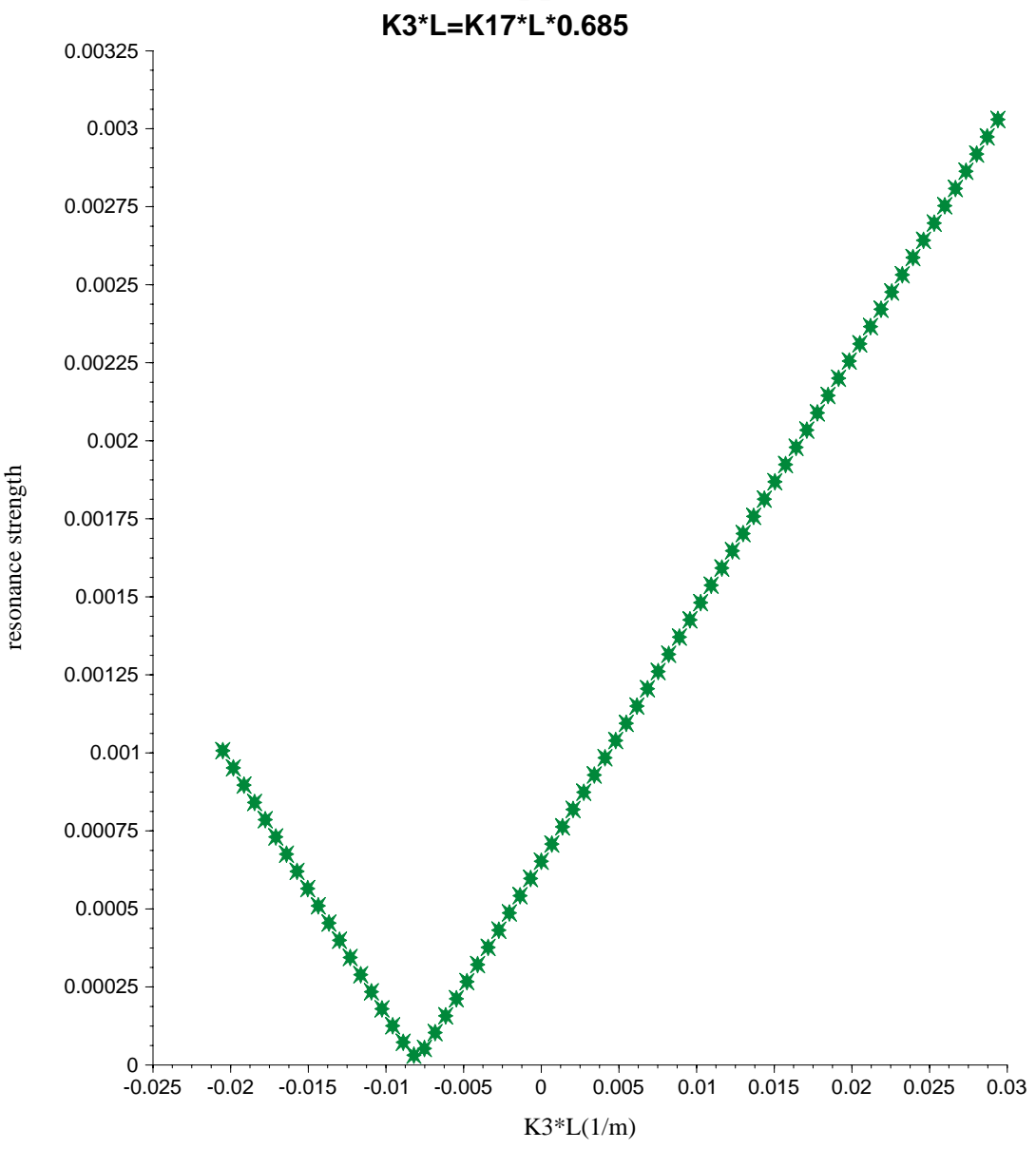


Figure 3: Resonance strength as determined by using equations $3.4-3.6$

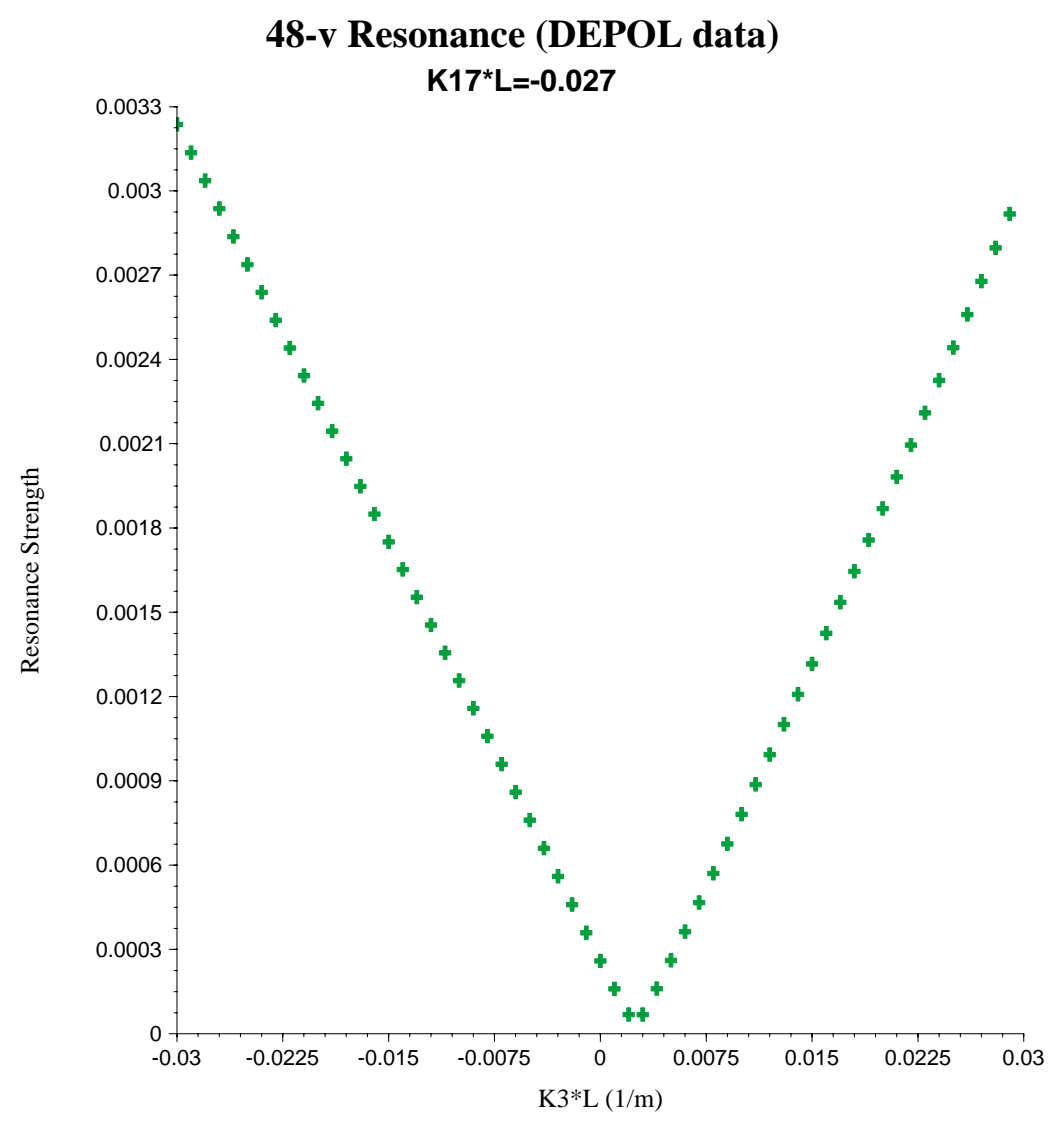


Figure 4: Resonance strength with quadrupoles operating in the $3 \mathrm{rd}$ and $17 \mathrm{th}$ positions. Data generated by DEPOL

\section{Tune and Betatron perturbations}

It is well known that the change in tune can be evaluated by:

$$
\Delta \nu=\frac{1}{4 \pi} \int \beta(s) k(s) d s
$$

Here $k(s)$ is the distributed quadrupole error which is the same as $g(s)$. The shift in tune for the two quadrupole case will be given by;

$$
\Delta \nu=\left(\beta_{1} g_{1}+\beta_{2} g_{2}+\beta_{3} g_{3}\right) \frac{P}{4 \pi}
$$

Unfortunately $\beta$ is not wholly independent of the integrated field strength. But for tune evaluations the change in $\beta$ at each quadrupole is not significant in the range of field strength we are interested in and equation 3.9 works very well. Still we are interested in understanding how our betatron function is altered around the whole lattice to understand the nature of our error in equation 3.2 better. It is well known that the betatron function can change according to.

$$
\begin{gathered}
J_{m}=\frac{1}{2 \pi} \oint \beta k(s) e^{-i m \varphi} d s \\
\frac{\Delta \beta(s)}{\beta(s)}=-\frac{\nu_{o}}{2} \sum_{m=-\infty}^{\infty} \frac{J_{p} e^{i m \varphi(s)}}{\nu_{o}^{2}-(m / 2)^{2}}
\end{gathered}
$$


It is however possible to evaluate these equations more explicitly for our case.

$$
J_{m}=\sum_{n=0}^{P-1} e^{-\frac{i 2 \pi n m}{P}} \frac{\beta_{1} g_{1} e^{-i m \varphi_{1}}+\beta_{2} g_{2} e^{-i m \varphi_{2}}}{2 \pi}
$$

Which when the sum is evaluated gives:

$$
J_{m}=e^{-\frac{i m(P-1) \pi}{P} \zeta_{P}\left(\frac{m}{P}\right)} \frac{\beta_{1} g_{1} e^{-i m \varphi_{1}}+\beta_{2} g_{2} e^{-i m \varphi_{2}}}{2 \pi}
$$

Now considering only when the enhancement function equals $P(-1)^{l}$ when $\mathrm{m}=\mathrm{lP}$ and $\mathrm{l}=$ integer. $(\mathrm{P}=$ even $)$

$$
J_{l P}=\frac{(-1)^{l P} P}{2 \pi}\left(\beta_{1} g_{1} e^{-i P l \varphi_{1}}+\beta_{2} g_{2} e^{-i P l \varphi_{2}}\right)
$$

Here we introduce $\beta^{(0)}$ to indicate the unperturbed betatron function. Finally inserting equation 3.14 into 3.11 and changing the sum from 0 to infinity we obtain

$$
\Delta \beta_{s}=\beta_{s}\left(g_{1} \beta_{1}^{(0)} \alpha_{s, 1}+g_{2} \beta_{2}^{(0)} \alpha_{s, 2}\right)
$$

Where we have introduced

$$
\alpha_{i, j}=\frac{-v_{0} P}{2 \pi} \sum_{l=0}^{\infty} \frac{\cos \left(l P\left(\varphi_{i}-\varphi_{j}\right)\right)}{v_{2}-\left(\frac{l P}{2}\right)^{2}}
$$

We note that $\alpha_{i, j}=\alpha_{j, i}$ because cosine is an even function. We also note that the subscript $s$ denotes where the Betatron perturbation is being evaluated. 


\section{Calculations for Three Families of Quadrupoles}

Clearly using two quadrupoles can give us arbitrary control over either the vertical or horizontal tunes. However in the particular instance of the $3 \mathrm{rd}$ and $17 \mathrm{th}$ quadrupole the requirement to both keep the tune fixed and fully suppress each resonance make fulfilling both requirements impossible since equation 3.6 and 3.7 fixes the field to values different than equation 3.9. Perhaps using three families of quadrupoles will allow the freedom necessary to satisfy both requirements. For this case we proceed first considering the change in the betatron function to be small. Thus we alter equation 3.9 to include a third quadrupole. Likewise we proceed with equations 3.3-3.5 including a third quadrupole. If we assume that our unperturbed resonance strength is composed primarily of a real component one can obtain the following fixed tune, zero resonance solution.

$$
\begin{gathered}
g_{3}=\frac{\frac{-R_{0} 4 \pi}{(1+G \gamma) P} \sqrt{\frac{\pi \gamma}{\epsilon_{N}}}+\frac{\Delta v 4 \pi\left(\sqrt{\beta_{2}} \cos \left(P N \varphi_{2}\right)-m_{2,1} \sqrt{\beta_{1}} \cos \left(P N \varphi_{1}\right)\right)}{P\left(\beta_{1} m_{2,1}-\beta_{2}\right)}}{\left(\frac{\beta_{3}-m_{3,1} \beta_{1}}{m_{2,1} \beta_{1}-\beta_{2}}\right)\left(\sqrt{\beta_{2}} \cos \left(P N \varphi_{2}\right)-m_{2,1} \sqrt{\beta_{1}} \cos \left(P N \varphi_{1}\right)\right)-m_{3,1} \sqrt{\beta_{1}} \cos \left(P N \varphi_{1}\right)+\sqrt{\beta_{3}} \cos \left(P N \varphi_{3}\right)}(3.17) \\
g_{1}=-g_{2} m_{2,1}-g_{3} m_{3,1} \\
g_{2}=g_{3}\left(\frac{\beta_{3}-m_{3,1} \beta_{1}}{m_{2,1} \beta_{1}-\beta_{2}}\right)-\frac{\Delta v 4 \pi}{P\left(\beta_{1} m_{2,1}-\beta_{2}\right)}
\end{gathered}
$$

Where we use

$$
m_{i, j}=\sqrt{\frac{\beta_{i}}{\beta_{j}}} \frac{\sin \left(P N \varphi_{i}\right)}{\sin \left(P N \varphi_{j}\right)}
$$

If our resonance is primarily imaginary then our solution is; 


$$
g_{3}=\frac{\frac{-R_{0} 4 \pi}{(1+G \gamma) P} \sqrt{\frac{\pi \gamma}{\epsilon_{N}}}+\frac{\Delta v 4 \pi\left(\sqrt{\beta_{2}} \sin \left(P N \varphi_{2}\right)-I_{2,1} \sqrt{\beta_{1}} \sin \left(P N \varphi_{1}\right)\right)}{P\left(\beta_{1} I_{2,1}-\beta_{2}\right)}}{\left(\frac{\beta_{3}-I_{3,1} \beta_{1}}{I_{2,1} \beta_{1}-\beta_{2}}\right)\left(\sqrt{\beta_{2}} \sin \left(P N \varphi_{2}\right)-I_{2,1} \sqrt{\beta_{1}} \sin \left(P N \varphi_{1}\right)\right)-I_{3,1} \sqrt{\beta_{1}} \sin \left(P N \varphi_{1}\right)+\sqrt{\beta_{3}} \sin \left(P N \varphi_{3}\right)}(3
$$

$$
\begin{gathered}
g_{1}=-g_{2} I_{2,1}-g_{3} I_{3,1} \\
g_{2}=g_{3}\left(\frac{\beta_{3}-I_{3,1} \beta_{1}}{I_{2,1} \beta_{1}-\beta_{2}}\right)-\frac{\Delta v 4 \pi}{P\left(\beta_{1} I_{2,1}-\beta_{2}\right)}
\end{gathered}
$$

Where we use

$$
I_{i, j}=\sqrt{\frac{\beta_{i}}{\beta_{j}}} \frac{\cos \left(P N \varphi_{i}\right)}{\cos \left(P N \varphi_{j}\right)}
$$

If we consider the first theta location to be the $3 \mathrm{rd}$, second the 15 th and third the 17th quadrupole family we can use these equations to estimate of the field strength necessary to keep $\Delta \nu_{z}$ zero while suppressing the $G \gamma=48-v$ resonance. Calculation shows $g_{3}=0.0057 g_{2}=-0.00667$ and $g_{1}=0.003945$. These clearly are reasonable field strength values. From these numbers we can also calculate the horizontal tune drift to be approximately 0.06. Using DEPOL however we found our actual field strength values to be $g_{3}=-0.014 g_{2}=0.006$ and $g_{1}=0.001$ here $\nu_{z}=8.7322$ and $v_{x}=8.4696$. Clearly our horizontal tune is not acceptable so if we relax our vertical tune requirements we can find several cases which accomplish our goal of both suppression and tune control. An example can be see in Figure 5. However a note should be made about this particular plot.

One might now wonder if the use of three quadrupoles would afford us the strength to suppress the strong resonances. A quick calculation shows though 
that the required field strength values remain impracticable. For example the $G \gamma=36-v$ resonance requires $g_{3}=0.206$ clearly beyond the capcity of the quadrupoles available.

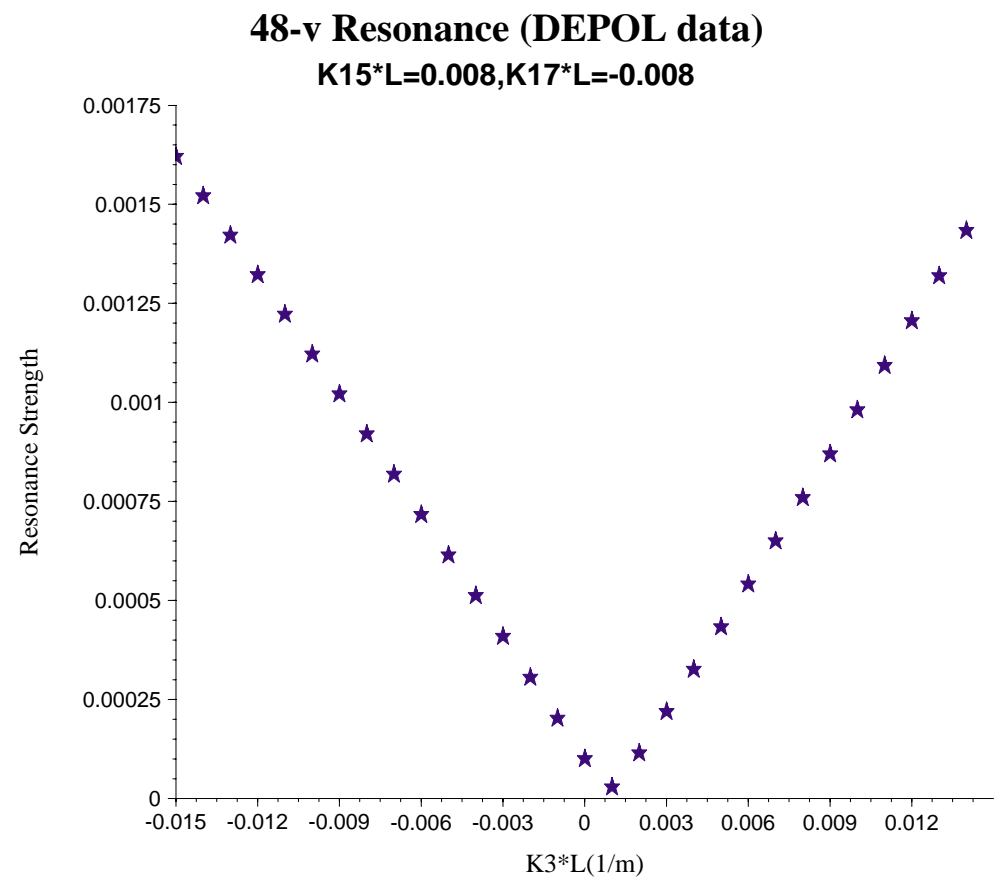

Figure 5: Using three quadrupole families at the $3 \mathrm{rd}, 15 \mathrm{th}$ and 17 th positions the resonances is both minimized and vertical and horizontal tunes kept within the integer and half integer stop-bands $\left(v_{x}=8.617 v_{z}=8.629\right)$.

The results for the weak resonance appear encouraging when considering the use of three quadrupoles. It appears that complete suppression of the resonance and 
maintaining the vertical and horizontal tune within the integer and half-integer stop bands is possible. But this requires the installation of quadrupoles in the fifteenth position. One might wonder if we move our goal from completely supressing the weak resonances to significantly reducing them, what the maximum reduction possible is using the existing $3 \mathrm{rd}$ and 17 th families while remaining with in the integer and half integer stop bands. Going ahead with an full field strength sweep for the 3rd and 17th families has shown that with $\nu_{x}=8.5216$, $\nu_{z}=8.844, g_{1}=-0.002$ and $g_{2}=-0.007$ we can achieve a $34.5 \%$ reduction in the resonance strength, this corresponds to a $2.4 \%$ depolarization. Similarly for $24+v$ at $v_{x}=8.501, v_{z}=8.886, g_{1}=-0.004$ and $g_{2}=-0.007$ we can achieve a $26 \%$ reduction in resonance strength, this corresponds to a $1.83 \%$ depolarization. Finally for $24-v$ at the same tune and field strength values as the $24+v$ case we can achieve a $53 \%$ reduction in resonance strength corresponding to a $0.17 \%$ depolarization. With this correction scheme would should be able to achieve about $4.34 \%$ depolarization from all the weak resonances this compares with a $9.4 \%$ depolarization on a bare machine. Of course we will have to back away from the half integer stop band so this represents only the upper limit of polarization gains.

\section{Correction to Quadrupole Resonance Perturbations}

Our approximation given in equation 3.2 while very close to actual perturbation values at each quadrupole, does not account for the significant effect which the perturbation to the betatron function will have on the original elements of the lattice. An examination of the calculated response of the resonance strength to varying quadrupole field strength showed a distinctly linear response. This is an encouraging prospect if one where interested in developing a more accurate linear model of this process. We proceed by noting that the resonance value 
calculated in Depole can be expressed as in a general simplified fashion.

$$
\varepsilon_{k}=\sum_{n} A_{n} \sqrt{\beta_{n}}
$$

Here $A_{n}$ is a function of the phase, field strength, and bending angle at each element. When one introduces a quadrupole element into the lattice, the betatron values at each element are perturbed in a manner described in equation 3.15 and 3.16. For a single quadrupole equation 3.35 can be expressed with a small deviation $\delta$ :

$$
\varepsilon_{k}+\delta=\sum_{n} A_{n} \sqrt{\beta_{n}+\Delta \beta_{n}}
$$

where,

$$
\begin{gathered}
\Delta \beta_{n}=\beta_{n} g_{1} \beta_{1} \alpha_{n, 1} \\
\varepsilon_{k}+\delta=\sum_{n} A_{n} \sqrt{\beta_{n}}\left(1+g_{1} \beta_{1} \alpha_{n, 1}\right)^{1 / 2}
\end{gathered}
$$

providing that $\left|g_{1}\right|<.1$ the square root can be expanded to first order and we arrive at an approximation for $\delta$ :

$$
\delta=g_{1} \beta_{1} \sum_{n} A_{n} \sqrt{\beta_{n}} \frac{\alpha_{n, 1}}{2}
$$

or more explicitly:

$$
\delta=\frac{-v_{0} P \beta_{1} g_{1}}{4 \pi} \sum_{n, l} A_{n} \sqrt{\beta_{n}} \frac{\cos \left(\varphi_{n}-\varphi_{1}\right)}{\nu_{0}^{2}-\left(\frac{l P}{2}\right)^{2}}
$$

An observation of the functional behavior reveals that $\delta$ is a function $g_{1}$ times 
a value constant for each resonance. So $\delta$ is clearly to first order a linear function of $g_{1}$. A direct calculation of equation 3.31 is not very practical since this is really what DEPOL already accomplishes but if we can establish the slope as a function of $g_{1}$ this makes subsequent evaluation of the resonance strength at each $g_{1}$ value much quicker and easier. So now our new improved resonance approximation becomes

$$
\varepsilon_{k}=R_{0}(N P)+\Delta \varepsilon\left(g_{1}, N P\right)_{k}+\delta\left(g_{1}, N P\right)
$$

where $\Delta \varepsilon_{k}$ was given in 3.2 . In principle we can factor out $g_{1}$ from the last two terms and fit the line simply using two data points generated by DEPOL. In the case when there are more than one quadrupole insertion the development is identical with each new quadrupole introducing a perturbation linearly dependent on its field strength. This confirms what saw visually in our Figures $2-5$. 


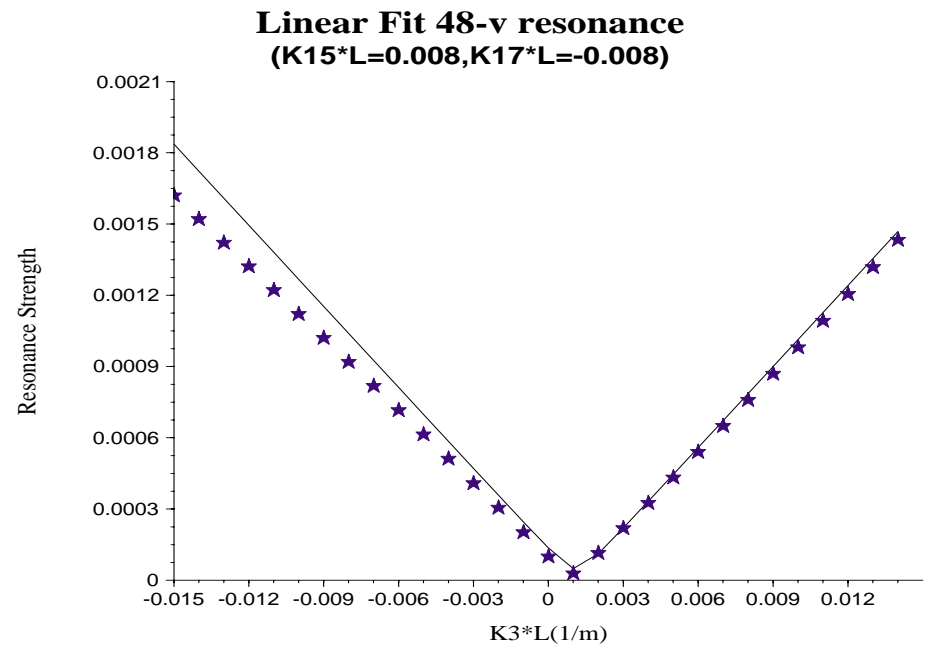

Figure 6: An overlay of our linear fit on data from Figure 5. 


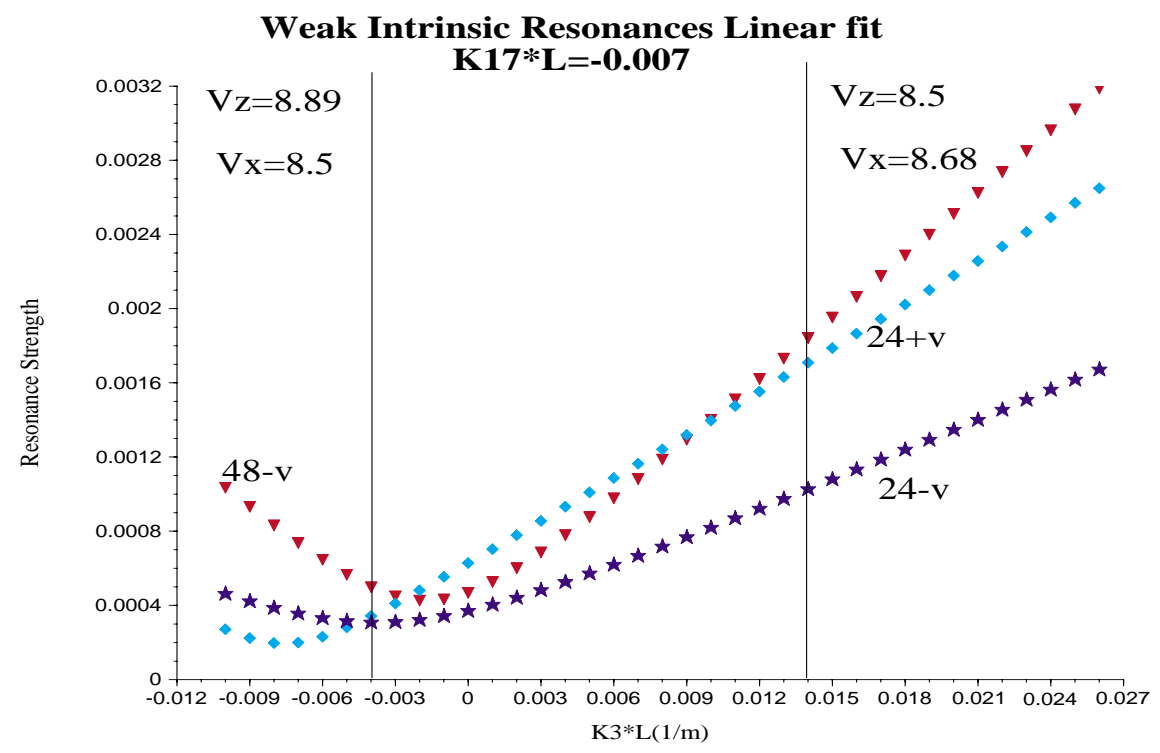

Figure 7: Locating the minimum possible resonance strength using using linear fit. Quadrupoles are located in the 3rd and 17th positions.Vertical lines indicate location of vertical and horizontal tune values

Below is the full three dimensional fit to our resonance using only two data points generated by DEPOL. The full complex function is:

$$
\begin{array}{r}
\varepsilon(24-v)_{k} \approx g_{1}(0.0048)-g_{2}(0.01253)+g_{3}(0.07153)+0.00024+ \\
i\left(-g_{1}(0.05513)+g_{2}(0.0272)+g_{3}(0.00906)+0.000011\right)
\end{array}
$$




$$
\begin{gathered}
\varepsilon(24+v)_{k} \approx g_{1}(0.06186)-g_{2}(0.01173)+g_{3}(0.0362)+0.000507+ \\
i\left(-g_{1}(0.04853)+g_{2}(0.02773)-g_{3}(0.006266)-0.000022\right) \\
\varepsilon(48-v)_{k} \approx g_{1}(0.06193)+g_{2}(0.02706)-g_{3}(0.05813)+0.000652+ \\
\quad i\left(g_{1}(0.0956)+g_{2}(0.01353)-g_{3}(0.0066)+0.000029\right)
\end{gathered}
$$

The tune can then be tracked just using equation 3.9 applied to the three quadrupole case:

$$
\begin{aligned}
& v_{z} \approx 8.73-\frac{3}{\pi}\left(g_{1}(22.164)+g_{2}(10.355)+g_{3}(22.016)\right) \\
& v_{x} \approx 8.69+\frac{3}{\pi}\left(g_{1}(10.403)+g_{2}(22.238)+g_{3}(10.454)\right)
\end{aligned}
$$

Using these equations we can completely map out the behavior of the resonances and tunes under the perturbing effect of a family of quadrupoles. Proceeding with this fit we can achieve a better approximation as show in Figure 6 repeating the $48-v$ resonance graph in Figure 5 . In Figure 7 we also repeat the results we found in the previous subsection. We show again just how far we can go in minimizing the weak resonances with our existing quadrupoles. 


\section{Technical Considerations regarding Quadrupole performance}

In an ideal situation we could move the field strength in the quadrupoles to any chosen value during acceleration. Unfortunately in reality we limited by the inductivity of the existing quadrupoles. This situation forces us to account for the tune requirements of the $\mathrm{rf}$ dipole. Injection occurs below the $G \gamma=0+v_{z}$ resonance and extraction just after the $G \gamma=36+v_{z}$ resonance. Since the resonance frequency of the rf dipole system must be set manually before a run, the operating tune is fixed at each strong intrinsic resonance. Currently the RF dipole has been set to a resonance frequency corresponding to a vertical tune of 8.7 and applied to the $G \gamma=0+v_{z}, G \gamma=12+v_{z}$, and the $G \gamma=36 \pm v_{z}$ resonances.

\begin{tabular}{|c|c|c|c|c|c|c|}
\hline $\bar{\gamma}$ & From $\mathrm{G} \gamma$ & To $\mathrm{G} \gamma$ & $\Delta G \gamma$ & $\Delta t(\mathrm{sec})$ & $\Delta I(\mathrm{Amps})$ & $\Delta \mathrm{K}^{*} \mathrm{~L}(1 / \mathrm{m})$ \\
\hline \hline 6.69 & $0+v_{z}^{*}$ & $24-v_{z}$ & 6.6 & 0.058 & 384 & 0.032 \\
\hline 10.04 & $24-v_{z}$ & $12+v_{z}^{*}$ & 5.4 & 0.048 & 318 & 0.018 \\
\hline 13.39 & $12+v_{z}^{*}$ & $36-v_{z}^{*}$ & 6.6 & 0.058 & 384 & 0.016 \\
\hline 16.73 & $36-v_{z}^{*}$ & $24+v_{z}$ & 5.4 & 0.048 & 318 & 0.0105 \\
\hline 20.08 & $24+v_{z}$ & $48-v_{z}$ & 6.6 & 0.058 & 384 & 0.0102 \\
\hline 23.43 & $48-v_{z}$ & $36+v_{z}^{*}$ & 5.4 & 0.048 & 318 & 0.0075 \\
\hline
\end{tabular}

Table 2: The maximum possible integrated field strength change between each resonance. We assume an acceleration of 4.8E-5 1/rad and employ a current transfer function of $1.73 \mathrm{E}-3 \mathrm{~T} / \mathrm{Amp}[10]$. The tune quadrupoles which are currently installed in the 3rd and 17th position in the AGS each have a total resistance of $\mathrm{R}=0.161 \Omega$, inductance of $\mathrm{L}=0.02567 \mathrm{H}$ and maximum Voltage of $\mathrm{V}=170 \mathrm{~V} .^{1}$ 
So if we keep the vertical tune at 8.7 and want to simultaneously suppress the weak intrinsic resonances we will have to jump back and forth between field field strengths necessary to suppress a particular weak resonance and values which fix the vertical tune at 8.7. For most of the resonance crossings this should not be a problem since the maximum change in field strength required to minimize a resonance is $\Delta g=-0.007$. Though the $48-v_{z}$ seems very close though in any event we will probably have to back away from the half integer resonance.

To apply this method it would be ideal if we could take very accurate data at two field strength points for each energy where we want to suppress a resonance. Unfortunately while this might be feasible at lower energies, at higher energies where our weak resonances are strongest we do not have the analyzing power to measure polarization to the accuracy required.

\section{Future Experiments}

The method can at most raise polarization by $10 \%$. However the error bar of the polarization measurement is $10 \%$ close to maximum energy of the AGS for a typical polarization measurements of 20-30 minutes. We could however test the working principle of this method by focusing on resonances at lower energies. If we look at the extreme cases our differential in polarization can be as much as $20 \%$. For example we could test the odd resonance at $G \gamma=12+v_{z}$, while we know that we cannot completely suppress this resonance we can strongly effect its polarization. Thus we can test in principle the workings of this method.

\footnotetext{
${ }^{1}$ The $(*)$ indicates the resonances where the RF dipole is employed.
} 


\section{References}

[1] A. Lehrach et al. , Suppressing intrinsic spin harmonics at the AGS, C$\mathrm{A} / \mathrm{AP} \# 11$ (2000).

[2] Ya.S. Derbenev, A.M. Kondratenko, Sov. Phys. Doklady 20, 562 (1976); Ya.S. Derbenev et al., Particle Accelerators 8, 115 (1978).

[3] M. Bai et al, Phys. Rev E56, 6002 (1997).

[4] M.Okamura, Partial Helical Snake, Int. Workshop on Polarized Protons at High $\mathrm{Q}^{2}$ region (2000), to be published.

[5] T.Roser, Proc. Workshop on Siberian Snakes and Depolarizing Techniques (1989) p.144.

[6] M.Bai ,T.Roser, Proceedings from 14th Int. Spin Phy. Symp, (2000), to be published.

[7] A. Lehrach, et al. Suppressing Instrinsic Spin Harmonics at the Cooler Synchrotron COSY, Nucl. Inst. Meth. A429, 26 (2000).

[8] S.Y.Lee , "Spin Dynamics and Snakes in Synchrotrons", World Scientific p.42 1997

[9] E.D. Courant and R.D. Ruth, The Acceleration of Polarized Protons in Circular Accelerators, BNL Note 51270 UC-28 (1980)

[10] E. Bleser, AGS Tune Quad Production Measurements, AGS/AD/Tech. Note No. 455,(1997) 